\title{
GTH 2019 in Berlin \\ Der Jahreskongress 2019 in Zahlen
}

"Science meets Clinical Practice" schrieben die Kongresspräsidenten Robert Klamroth und Jürgen Koscielny auf die Fahnen des 63. Jahreskongresses der GTH. Vom 27. Februar bis 2. März folgten rund 1.250 Gäste der Einladung in
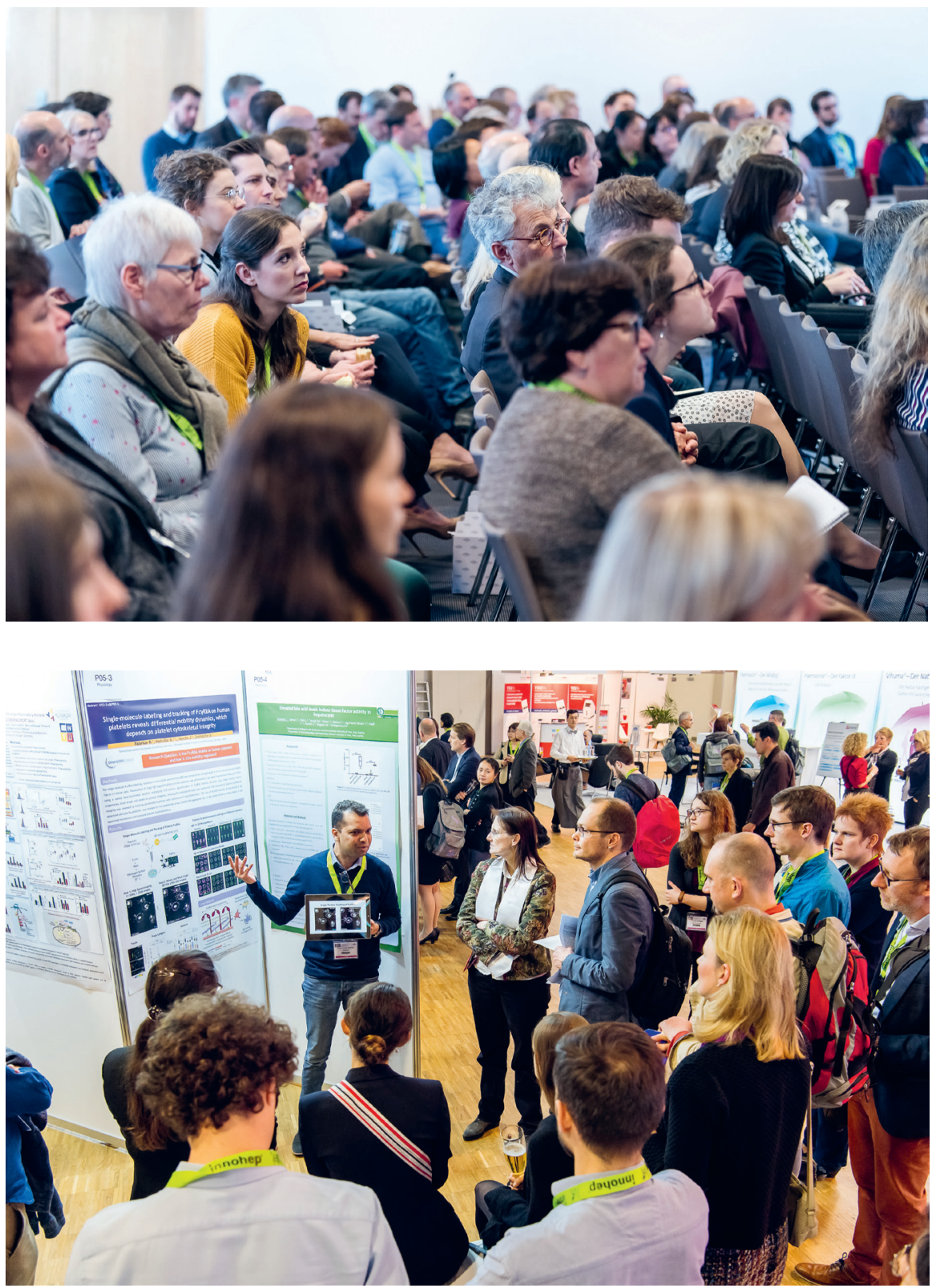
aus der Schweiz und Österreich und 53 internationale Besucher

Des Weiteren waren 511 registrierte Teilnehmer der Industrie, die bisher das Berliner Estrel Congress Center, davon fast 1.000 aus Deutschland, je 100 höchste registrierte Anzahl auf einem Jahreskongress der GTH, und erstmals weitere 312 externe ärztliche Teilnehmer am Fortbildungstag am 02. März auf dem diesjährigen Jahreskongress anwesend.
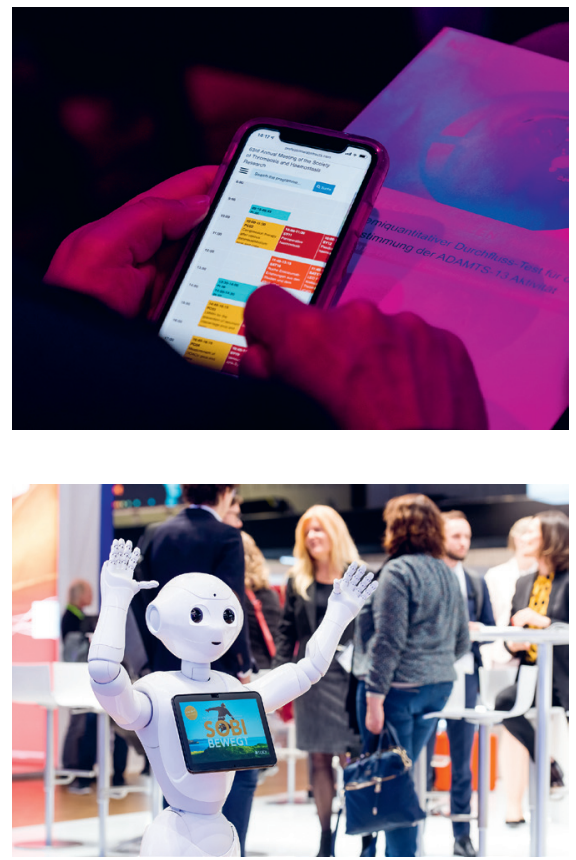

An den vier Kongresstagen fanden sieben Plenary Lectures statt sowie an jedem Tag parallel bis zu zehn Sessions. Die Posterausstellung umfasste in diesem Jahr 113 Poster. Als besonderes Event wurden zwei geführte Posterbegehungen mit Bewirtung angeboten, die von den Teilnehmern gerne angenommen wurden.

Auf den mehr als 1.600qm Industrieausstellung stellten 44 Aussteller aktuellste Medizinprodukte und-technik vor, weitere Informationen dazu gab es in den 21 Industriesymposien. 


\section{Eröffnung des 63. GTH Jahreskongresses in Berlin und Vergabe der Preise und Stipendien 2019}

Rund 450 Gäste nahmen an der Opening Ceremony am 27. Februar teil, in deren Rahmen jährlich vom Vorstand die Preise und Stipendien der GTH verliehen werden. Durch das Programm führten die GTH Vorsitzende Bettina Kemkes-Matthes sowie die Kongresspräsidenten Robert Klamroth und Jürgen Koscielny. Das Rahmenprogramm wurde musikalisch gestaltet vom Duo "Just two Cellos" mit Christoph Lamprecht und Christian Raudszus. Jürgen Koscielny führte bei der Eröffnungsfeier in seinem Vortrag durch die beeindruckende und bewegte Historie und Entwicklung der Berliner Charité.
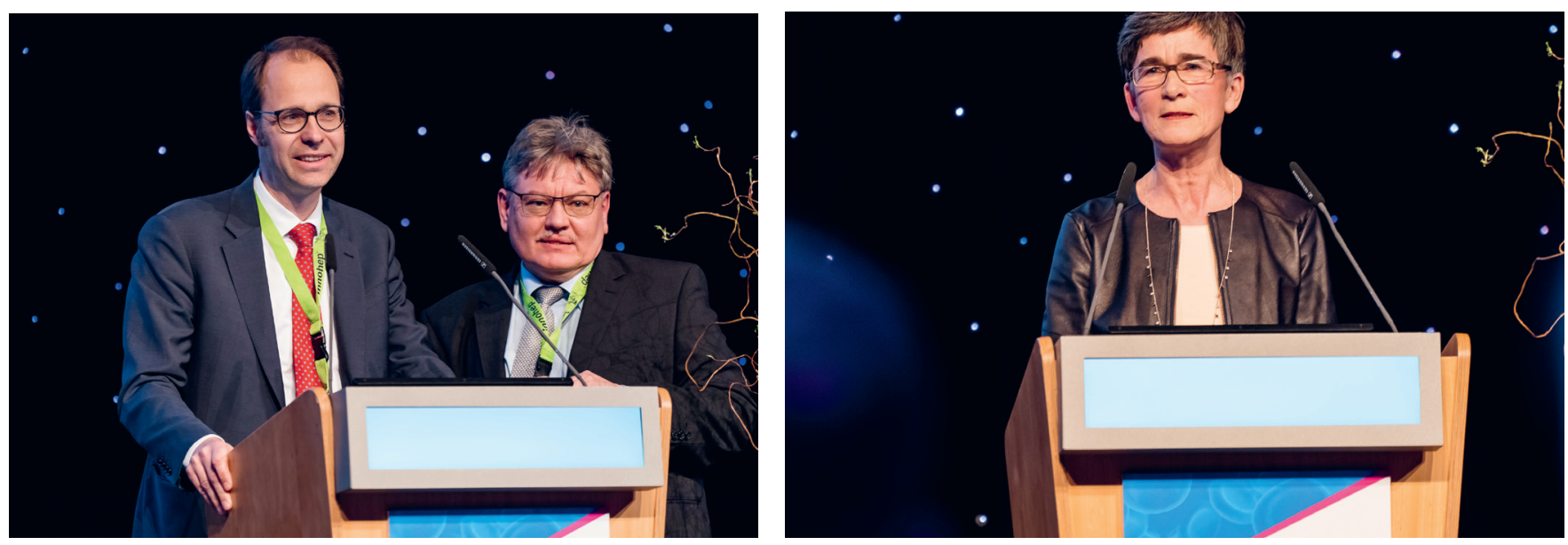

Die Kongresspräsidenten Robert Klamroth und Jürgen Koscielny und die Vorsitzende Bettina Kemkes-Matthes eröffneten den 63. Jahreskongress der GTH in Berlin.

Alexander-Schmidt-Preis Vorlesung Dr. Tobias Fuchs erhielt 2018 in Wien den Alexander-Schmidt-Preis. Er berichtete in einem spannenden Vortrag zum Thema Neutrophil Extracellular Traps (NETs) über seine Forschungen aus dem vergangenen Jahr.

\section{Alexander-Schmidt-Preis}

Für hervorragende Arbeiten auf dem

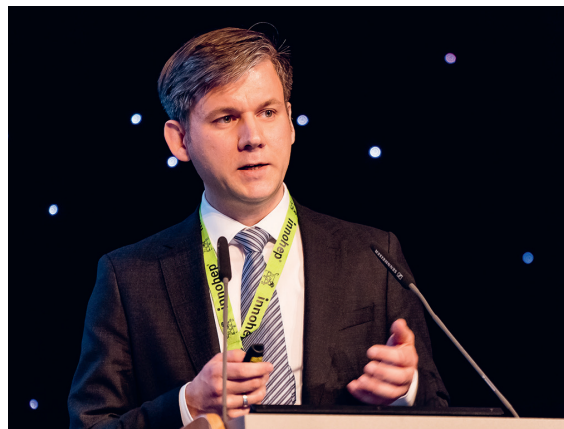

Tobias Fuchs, Alexander-Schmidt-Preisträger 2018, referierte zum Thema NETs.
Gebiet der Hämostaseologie vergibt die GTH jährlich diesen mit $15.000 €$ dotierten Preis. Bewerben können sich GTH Mitglieder mit hochrangigen Arbeiten aus dem Gebiet der Hämostaseologie / vaskulären Medizin, die von einem Gutachtergremium für die Veröffentlichung in einer wissenschaftlichen Zeitschrift angenommen oder bereits veröffentlicht worden sind.

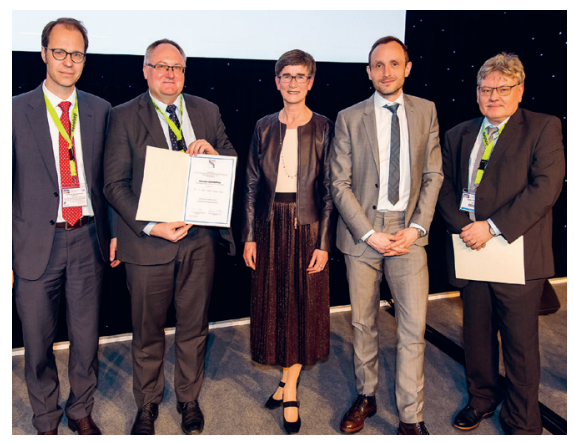

Stellvertretend für Katrin Czogalla-Nitsche, die leider nicht persönlich anwesend sein konnte, nahm Herr Oldenburg den Preis entgegen.
Der Alexander-Schmidt-Preis geht an: Dr. Katrin Czogalla-Nitsche aus Bonn für die Arbeit:

Warfarin and vitamin $\mathrm{K}$ compete for binding to Phe55 in human VKOR (Natural Structural \& Molecular Biology, Vol. 24, Nr. 1, Jan. 2018)

\section{Nachwuchsförderpreise}

Jährlich vergibt die GTH zwei mit je $2.500 €$ dotierte Förderpreise für hervorragende wissenschaftliche Arbeiten an junge Wissenschaftler aus den Gebieten Blutungskrankheiten und Thromboseforschung / vaskuläre Medizin, die von einem Gutachtergremium für die Veröffentlichung in einer wissenschaftlichen Zeitschrift angenommen oder bereits veröffentlicht worden sind.

Der Nachwuchsförderpreis Blutungskrankheiten konnte in diesem Jahr leider nicht vergeben werden, da keine Bewerbungen vorlagen. 


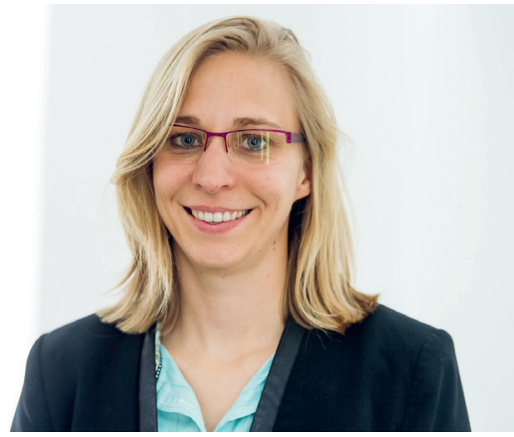

Ella Grilz aus Wien, die diesjährige Preisträgerin des Nachwuchsförderpreises Thromboseforschung.

Der Nachwuchsförderpreis Thromboseforschung/vaskuläre Medizin geht an: Dr. Ella Grilz aus Wien für die Arbeit: Frequency, risk factors and impact on mortality of arterial thromboembolism in patients with cancer (Haematologica, 2018; 103(9); 1549-1556)

\section{Hans-Egli-Forschungsstipendium}

Das mit 30.000€ ausgestattete Stipendium soll jungen Wissenschaftlern ermöglichen, sich für ein halbes Jahr intensiv der Forschung eines Projektes auf dem Gebiet der angeborenen Blutungskrankheiten zu widmen.

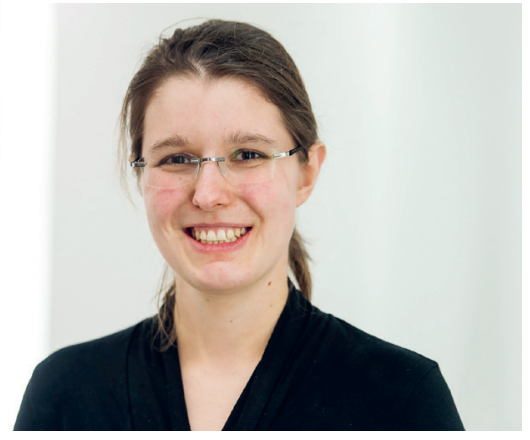

Anja Schmidt freut sich über das Hans-EgliForschungsstipendium.

\section{Das Hans-Egli-Forschungsstipendium geht an:}

Dr. Anja Schmidt aus Oberursel für das Projekt:

Zytokinprofile nach FVIII- und FIX-Stimulation - Relevanz für die unterschiedlichen Immunantworten?

\section{Rudolf-Marx-Stipendium}

Für maximal 25.000€ wird im Rahmen dieses Stipendiums ein umrissenes Forschungsprojekt aus dem Bereich der Hämostaseologie oder der vaskulären Biologie und Medizin an einer externen Forschungseinrichtung im In- oder Ausland gefördert.

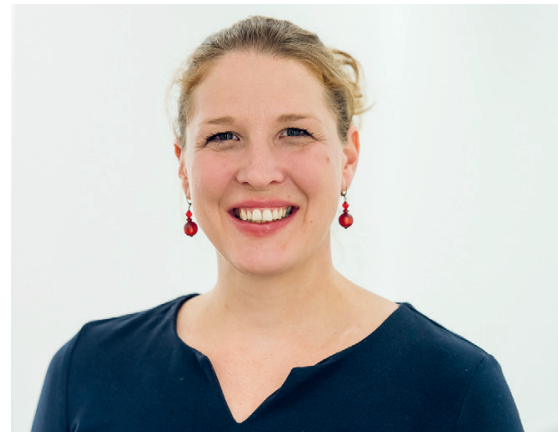

Johanna Gebhardt erhielt in diesem Jahr das Rudolf-Marx-Stipendium. Sie hat besonderen Grund zur Freude, denn erst im vergangenen Jahr bekam Sie in Wien das Hans-Egli Forschungsstipendium.

\section{Das Rudolf-Marx-Stipendium geht an:}

Dr. Johanna Gebhardt aus Wien für das Projekt:

Autoantibodies in primary ITP: Ability for platelet desialylation and not glycoprotein-specificity alters responsiveness to different treatment strategies and the bleeding phenotype.

\section{Die GTH Geschäftsstelle}

In diesem Jahr war der Stand der GTH Geschäftsstelle mit den aktuellen Medien der GTH und der GTH Akademie im Foyer neben der Registrierung zu finden.
Seit 2017 übernimmt die Geschäftsstelle mit Sitz in Köln Aufgaben wie Pflege und Strukturierung der Mitgliederdaten, Unterstützung des Vorstands, Öffent-

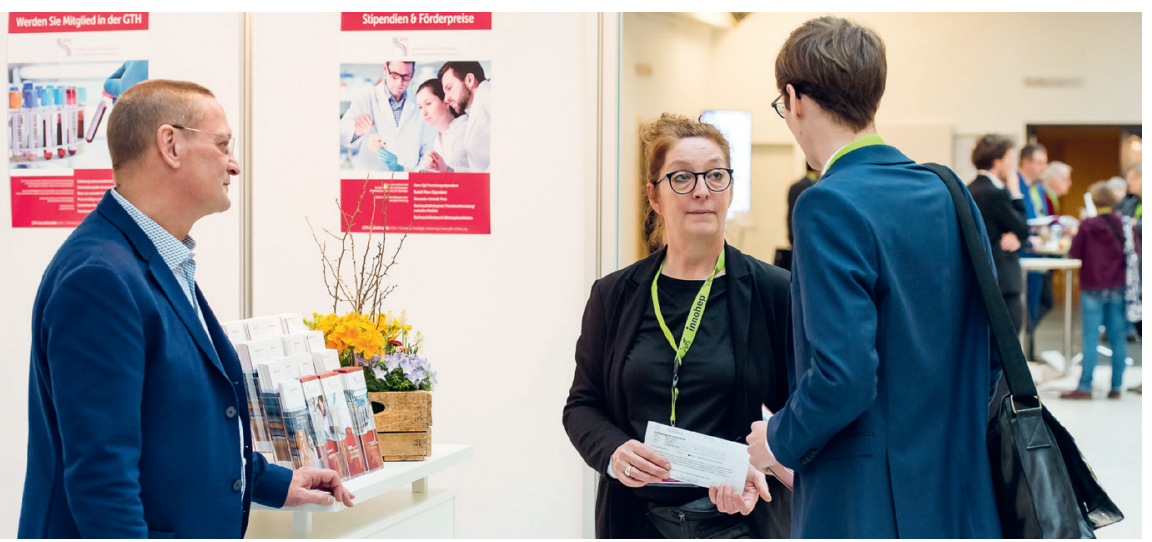

lichkeitsarbeit, Buchführung u.v.m. Inzwischen wurde auch die Webseite der GTH neu organisiert und ist in innovativem Design unter www.gth-online.org zu erreichen. Die interne und externe Kommunikation wurde z.B. durch regelmäßige, aktuelle Newsletter und etliche Printmedien intensiviert. Ganz neu hinzu kam die Organisation der GTH Akademie: So fanden unter dem Dach der Akademie im März erstmals die „Highlights 2019“ in Hannover statt, die von der Geschäftsstelle gemeinsam mit Vorstand, Wissenschaftlicher Leitung und Steering Committee realisiert wurden. Der Stand der Geschäftsstelle verzeichnete daher in 2019 eine Vielzahl von Besuchern mit den unterschiedlichsten Anliegen.

Mark Kayser und Kerstin Strohn, Mitarbeiter der GTH Geschäftsstelle in Köln, im Gespräch am Stand der GTH. 


\section{Mitgliederversammlung der GTH 2019 in Berlin}

In diesem Jahr durfte Prof. Bettina Kemkes-Matthes letztmalig als Vorstandsvorsitzende 154 Anwesende zur Mitgliederversammlung begrüßen. Neben einer umfangreichen Agenda erwarteten die GTH Mitglieder natürlich mit Spannung die satzungsgemäß alle vier Jahre stattfindenden Vorstandswahlen.

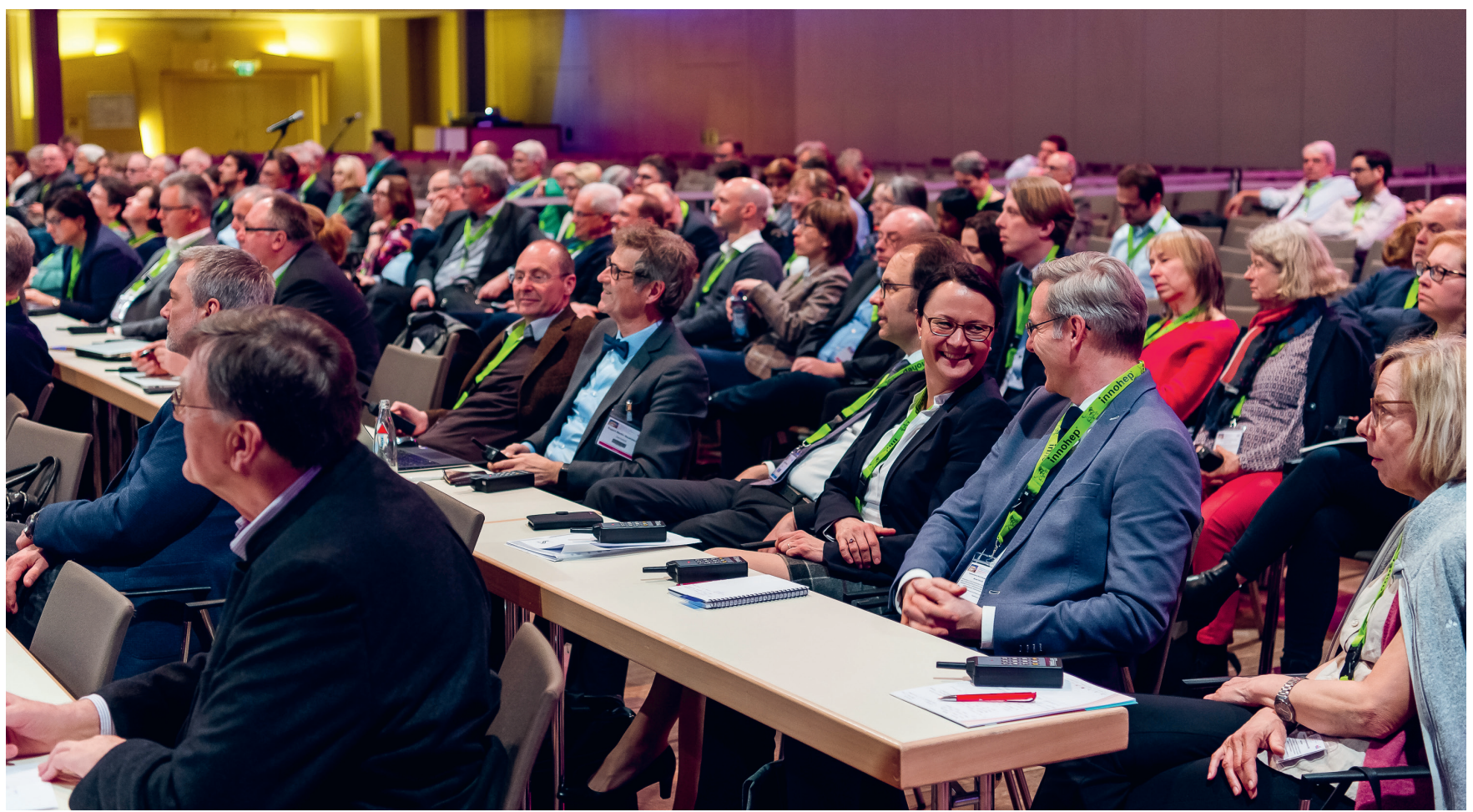

Frau Kemkes-Matthes blickte in Ihrem Bericht als Vorsitzende zurück auf die vergangenen vier Jahre und bedankte sich bei den Vorstandsmitgliedern für die konstruktive Arbeit: Schwerpunkte der Vorstandstätigkeiten waren der Aufbau der GTH Geschäftsstelle sowie der neu gegründeten GTH Akademie. Beides konnte erfolgreich etabliert werden. Besonders erfreulich sind die steigenden Mitgliederzahlen der GTH inzwischen 1030 - und die Realisierung der neuen Webseite. Weitere wichtige Themen waren die finale Fassung der (Muster-)Weiterbildungsordnung (MWBO), die enge Zusammenarbeit mit der European Thrombosis and Haemostasis Alliance (ETHA) sowie weiterhin die konzertierte Aktion mit dem Berufsverband der Deutschen Hämostaseologen (BDDH e.V.), denn inzwischen haben über 20 Zentren die Verträge mit den Krankenkassen unterschrieben.
Schriftührer Andreas Tiede berichtete detaillierter über die inzwischen zweijährige Zusammenarbeit mit der Kölner GTH Geschäftsstelle sowie über die im vergangenen Jahr gegründete GTH Akademie.

\section{GTH Geschäftsstelle}

Die Organisation und Verwaltung der Fachgesellschaft konnte durch die Geschäftsstelle auf ein professionelles Level gehoben werden. Viele Projekte wurden gemeinsam realisiert, unter anderem:

- Neuorganisation der Mitgliederdaten

- $\quad$ Überarbeitung von Verträgen mit Dienstleistern und entsprechende finanzielle Einsparungen

- $\quad$ Regelmäßige Newsletter zur Mitgliederinformation und-kommunikation

- Realisierung der GTH Akademie
GTH Akademie

Aktuell finden unter dem Dach der Akademie jährlich drei Fortbildungsveranstaltungen statt:

- Intensivkurs - Einstieg in die Hämostaseologie, Wiss. Leitung Bettina Kemkes-Matthes

- Laborkurs - Methoden der Hämostaseologie, Wiss. Leitung Ute Scholz, Organisation in Kooperation mit dem BFSH e.V.

- Highlights - Aktuelle Entwicklungen in der Hämostaseologie, Wiss. Leitung Andreas Tiede, Organisation in Kooperation mit der GTH Geschäftsstelle

Die Schatzmeisterin Edelgard Lindhoff-Last stellte den Jahresbericht der Gesellschaft vor und zeigte sich erfreut über die gute Bilanz sowie die gestiegene Zahlungsmoral. Hier zeigt das Beitrags- und Erinnerungsverfahren der Geschäftsstelle erste Früchte. 


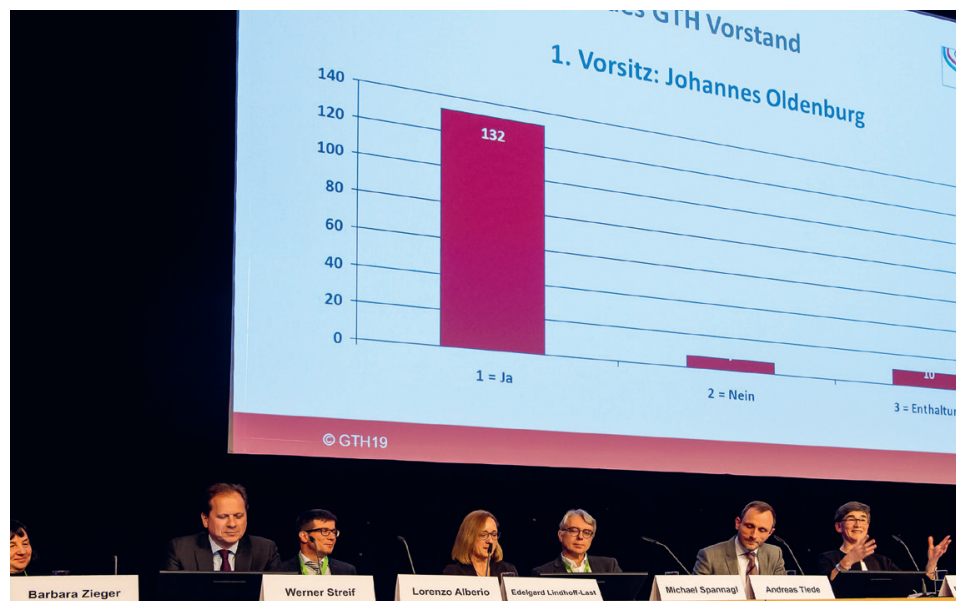

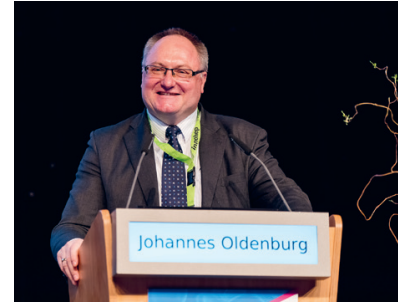

Vorstandsvorsitzender Prof. Dr. med. Johannes Oldenburg Vorsitzender und Direktor Universitätsklinikum Bonn

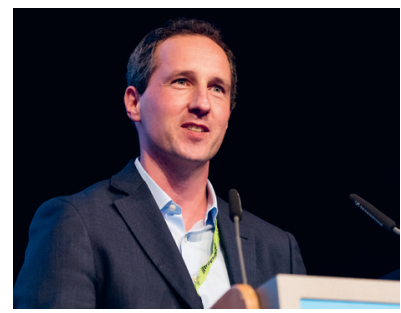

\section{Schriftführer}

Prof. Dr. med. Florian Langer

Universitätsklinikum

Hamburg-Eppendorf

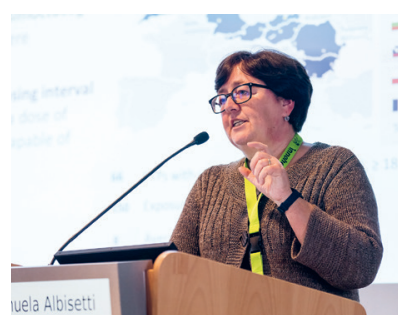

\section{Beisitzerin}

Prof. Dr. med. Manuela Albisetti Universitäts-Kinderspital Zürich

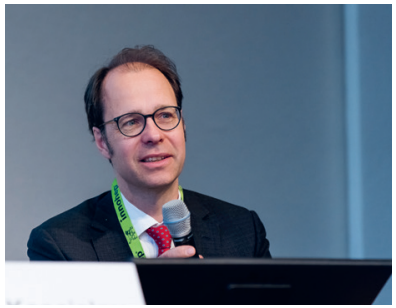

Stellv. Vorsitzender PD Dr. med. Robert Klamroth Vivantes Klinikum im Friedrichshain Berlin

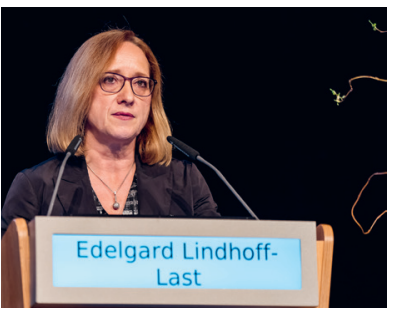

Schatzmeisterin

Prof. Dr. med. E. Lindhoff-Last

CCB Cardioangiologisches Centrum Bethanien Frankfurt

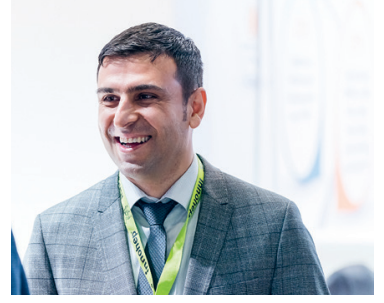

\section{Beisitzer}

Assoc. Prof. PD Dr. Cihan Ay Medizinische Universität Wien

\section{Der neue} Vorstand der GTH

Bei der diesjährigen Mit-

gliederversammlung standen satzungsgemä $\beta$ sechs der sieben Ämter des GTH Vorstands zur Wahl. Die Schatzmeisterin Edelgard Lindhoff-Last bleibt noch ein weiteres Jahr im Amt. Nach der Wahl per TED System stellen wir hier den neuen Vorstand vor:

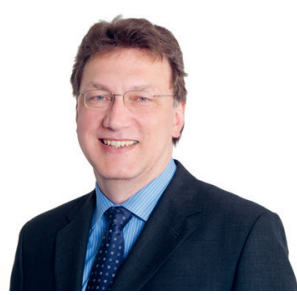

3. Beisitzer

Prof. Dr. med. Wolfgang Korte Zentrum für Labormedizin St. Gallen 


\section{Ständige Kommissionen und Arbeitskreise}

Die Ständigen Kommissionen und $\mathrm{Ar}$ beitskreise berichteten über den Fortschritt ihrer Arbeiten. Auf der Webseite der GTH finden Sie detaillierte Informationen sowie Ansprechpartner und Kontaktadressen. Sowohl mit fachlichen Fragen als auch zur Kooperation und Mitarbeit können sich Interessierte jederzeit gerne an die genannten Kollegen wenden.

\section{Der 24. Intensivkurs für klinische} Hämostaseologie 2018

Mit 86 Teilnehmern aus fünf Ländern war der Intensivkurs 2018 hervorragend besucht. Sowohl die Bewertungen als auch das finanzielle Ergebnis sind erfreulich. Wie in jedem Jahr erhielten die besten Teilnehmer der Abschlussklausur die kostenlose Teilnahme am Jahreskongress. Die Anmeldung zum Intensivkurs 2019 hat bereits begonnen, Ansprechpartnerin ist Susanne Rossi. Mehr Infos finden sich unter:

www.gth-intensivkurs.org

Da die Teilnehmerzahl begrenzt ist, sollten sich Interessierte rechtzeitig anmelden.

\section{GTH Jahrestagungen}

\section{Bremen 2020}

Der 64. Jahreskongress der GTH wird in

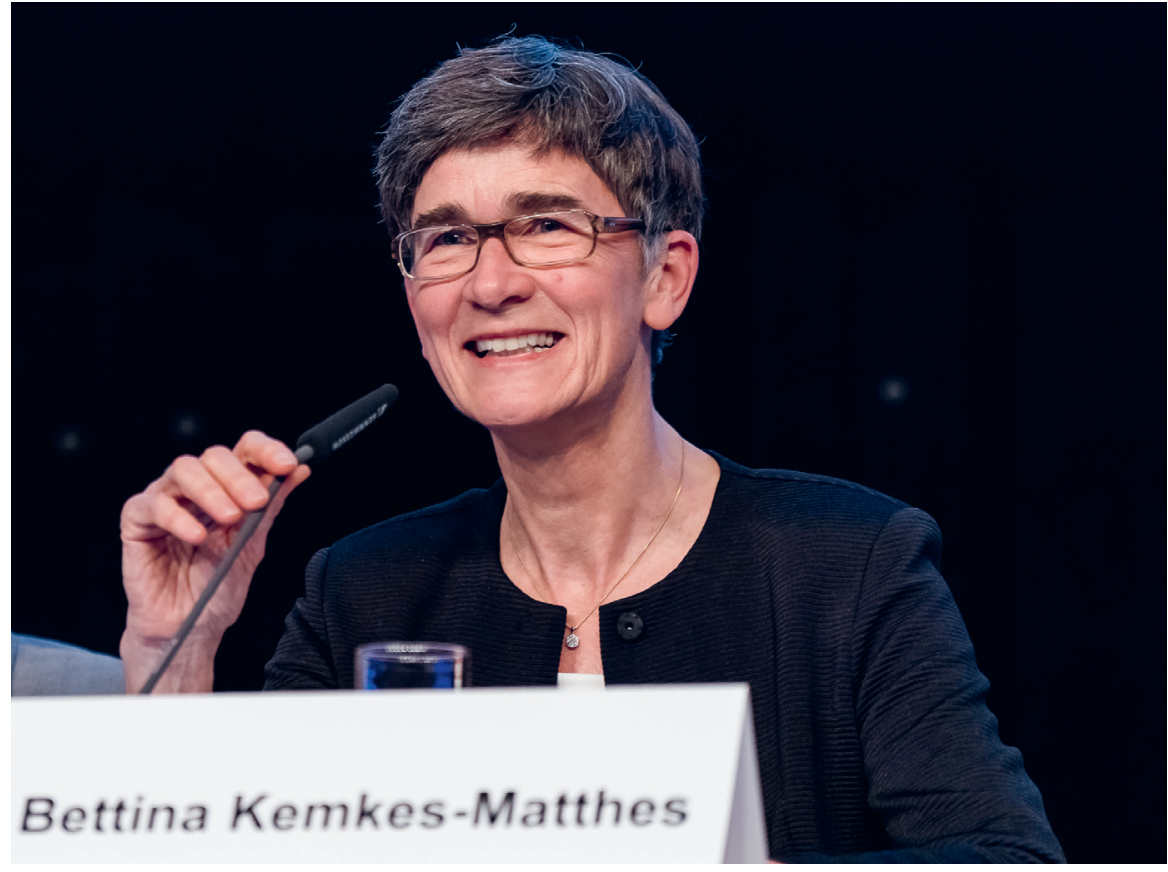

Bettina Kemkes-Matthes führte als Vorsitzende ein letztes Mal durch die Mitgliederversammlung.

Bremen stattfinden. Die Kongresspräsidenten Thomas Renné und Florian Langer berichteten zum Wechsel des Kongressortes von Hamburg nach Bremen.

\section{Lausanne 2021}

Der Kongresspräsident Lorenzo Alberio berichtete zu Location und Programm.

\section{Jahreskongress 2022}

Mehrheitlich wurde Sachsen mit den
Kongresspräsidenten Ute Scholz und Jan Beyer-Westendorf zum Tagungsort 2022 gewählt, die Stadt wird noch bekannt gegeben.

Das vollständige Protokoll der Mitgliederversammlung wird Anfang 2020 an dieser Stelle publiziert und geht den GTH Mitgliedern mit der Einladung zur nächsten Mitgliederversammlung zu.

\section{Special Guests der Charité}

Eine Delegation studierter mexikanischer Pflegefachkräfte unter Leitung von Dr. Beatrice Moreno folgte der Einladung der Kongresspräsidenten und nutzte die Möglichkeit zum Besuch des Jahreskongresses. Die Gruppe bereitet sich in der Charité auf Ihre Anerkennung vor und einige Teilnehmer interessieren sich insbesondere für eine Weiterbildung im Bereich Gerinnung. Die Studenten besuchten unter anderem den GTH Stand um sich über die Fachgesellschaft zu informieren.

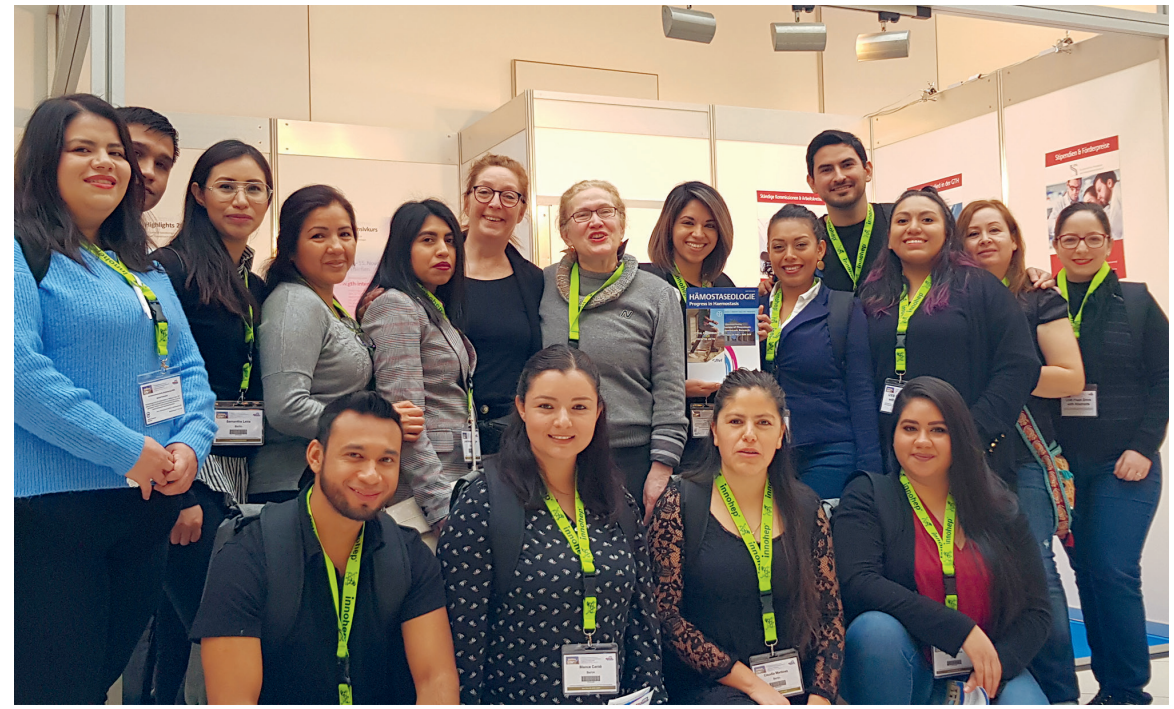




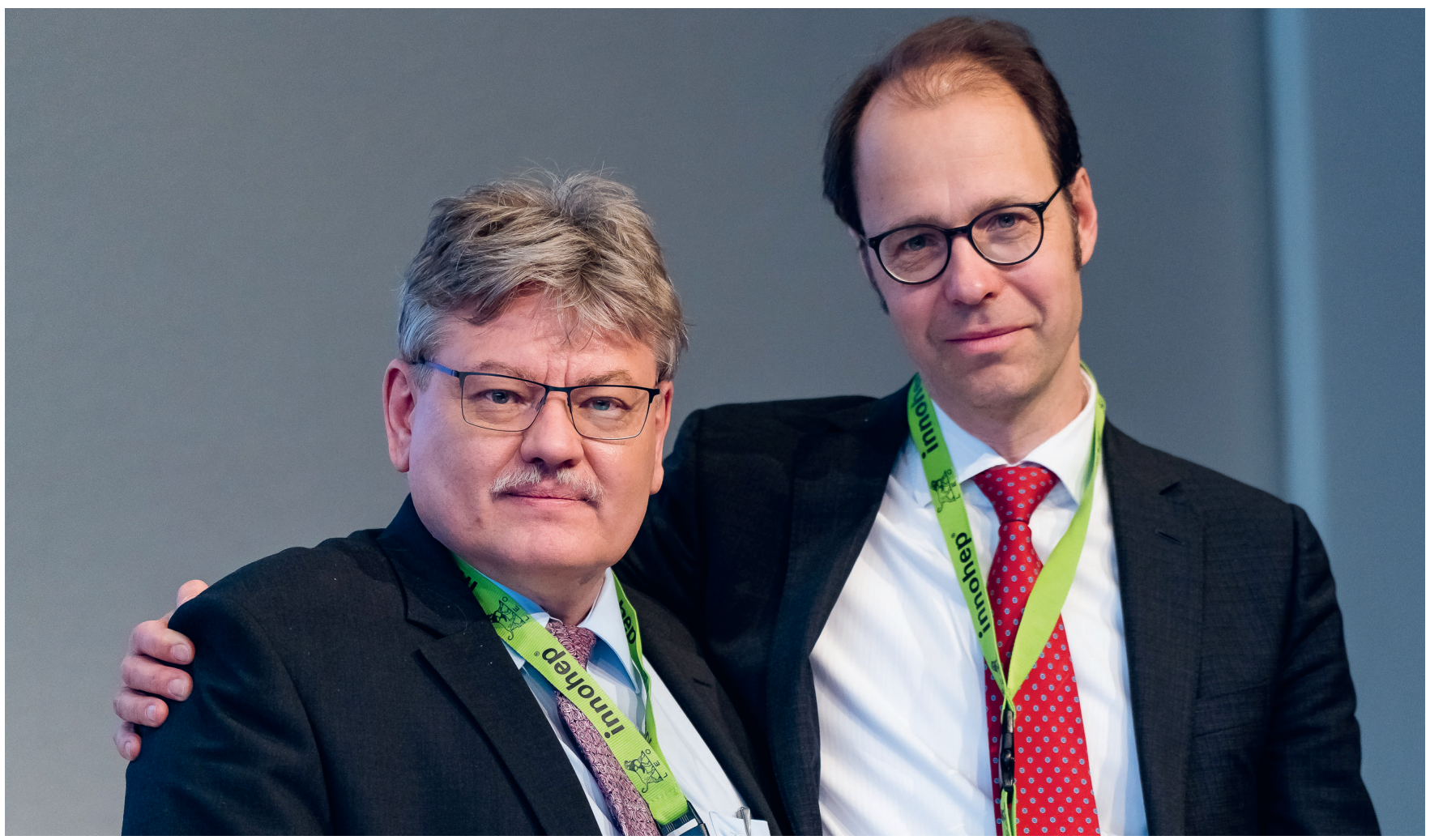

\section{Das Beste kommt zum Schluss}

\section{"Best of" des Jahreskongresses, Posterpreise und Stabsübergabe}

Zum Ende des Educational Day am 2. März verabschiedeten die Kongresspräsidenten Robert Klamroth und Jürgen Koscielny mit einer Mischung aus Freude, Wehmut und Erleichterung die Teilnehmerinnen und Teilnehmer der GTH und des Fortbildungstages.

Unter dem Motto „Das Beste kommt zum Schluss" wurden anschließend die Höhepunkte aus den wissenschaftlichen Vorträgen der vergangenen Tage in kompakten Referaten zusammengefasst:

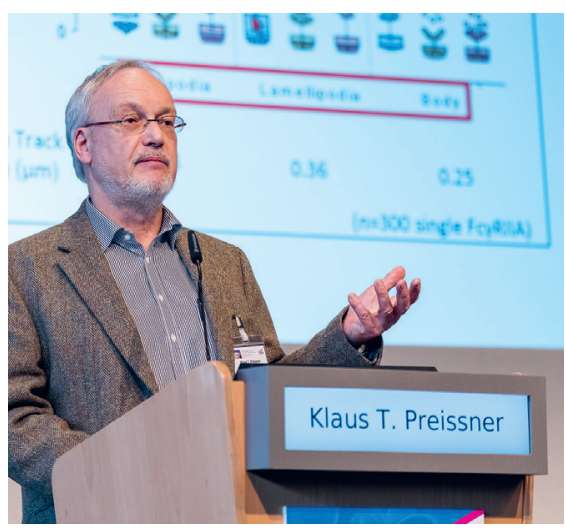

Prof. Dr. Klaus T. Preissner stellte die sieben besten Abstracts aus der Grundlagenforschung vor, Prof. Dr. Bernd Pötzsch fasste die Highlights zum Thema „Klinische Arbeit“ zusammen und Prof.

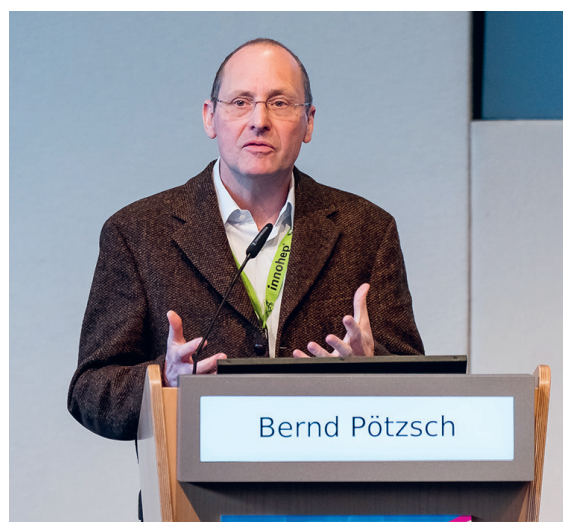

Christoph Male referierte zu den wichtigsten Themen aus der Pädiatrie.

\section{Posterpreise}

Im Anschluss fand die Preisverleihung

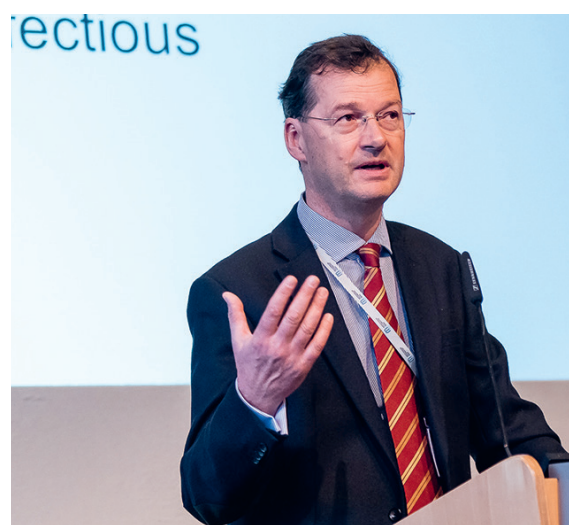

Prof. Dr. Klaus T. Preissner, Prof. Dr. Bernd Pötzsch und Prof. Christoph Male fassten die jeweils besten Referate und wichtigsten Themen aus den Bereichen Grundlagenforschung, Klinische Arbeit und Pädiatrie zusammen. 
für die besten Poster statt, ausgezeichnet wurden:

- $\quad$ Arbeitsgruppe von Prof. Dr. U. Sachs (Gießen, Marburg): Fetal and neonatal alloimmune thrombocytopenia (FNAIT): Evidence that placental rather than systemic inflammation is a modulator of disease severity

- $\quad$ Frau Dr. M. Thakur (München, Foto) für die Arbeitsgruppen aus München, Düsseldorf und Maastricht: Pancreatic tumor microvesicles invade immune cells via CD36 and distinctly promote tumor-associated deep vein thrombosis

- Herr Dr. O. Andres (Würzburg) für die THROMKIDplus Studiengruppe der GPOH und GTH: Use of targeted high-throughput sequencing for genetic classification of patients with bleeding diathesis and suspected platelet disorder

Im Rahmen der traditionellen Übergabe des Staffelstabes lud Thomas Renné stellvertretend für die beiden Kongresspräsidenten zum GTH Jahreskongress 2020 nach Bremen ein, wo ausgewählte Speaker über ihre eng mit der Hämostaseologie und der GTH verbundene Lebensgeschichte berichten werden.

Schlussendlich freuten sich Jürgen Koscielny und Robert Klamroth über ihren Status als „Ex-Präsidenten“ und wünschten ihren Nachfolgern Erfolg, „Geduld und Spucke".

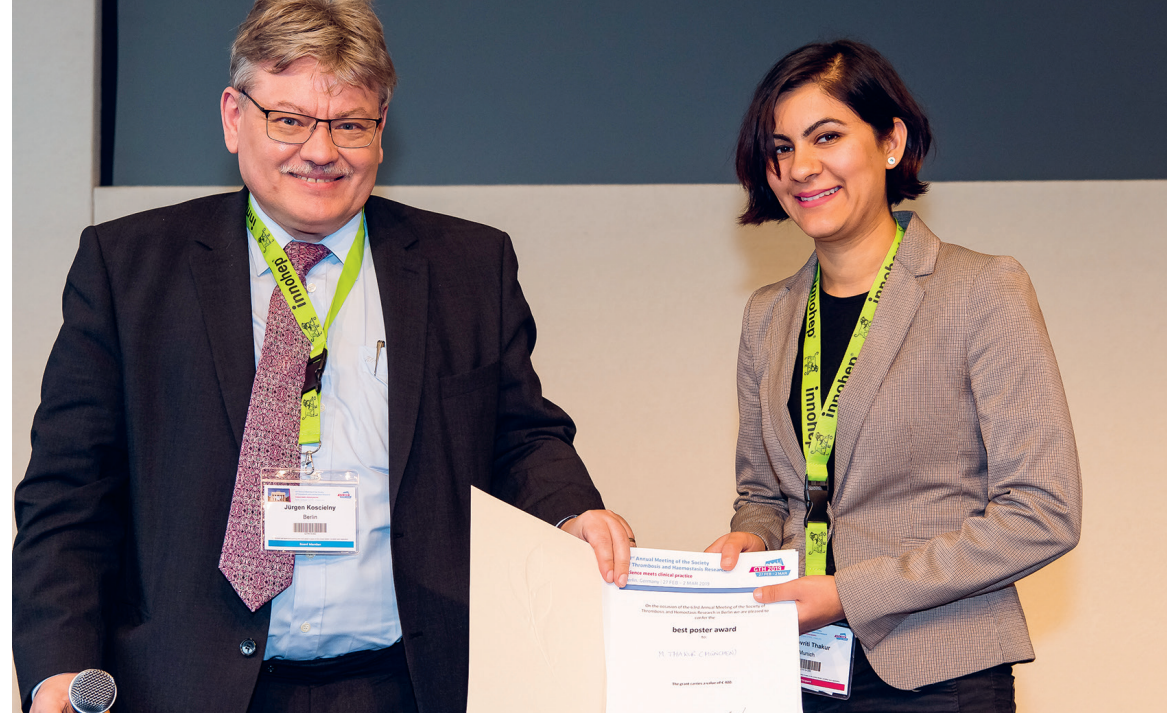

Dr. M. Thakur aus München nahm einen der drei Posterpreise von Jürgen Koscielny entgegen.

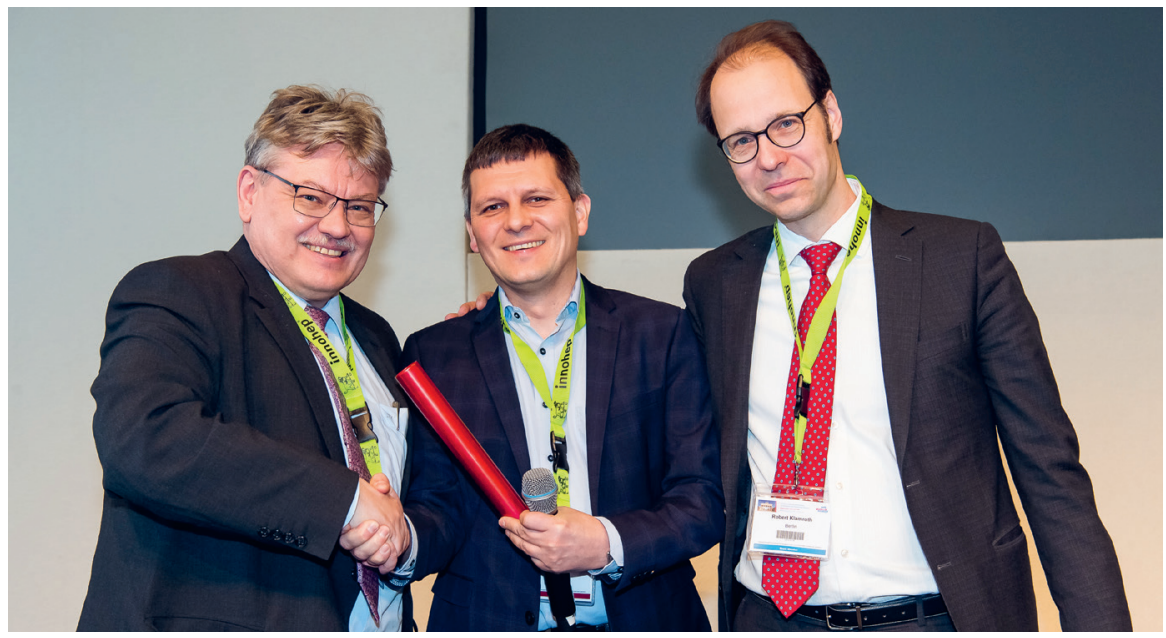

Finale des Jahreskongresses 2019: Der Stab liegt nun in den Händen von Thomas Renné (Mitte) und Florian Langer als Kongresspräsidenten 2020, mit besten Wünschen übergeben von Jürgen Koscielny (links) und Robert Klamroth (rechts).

\section{Termine für die kommenden GTH Jahreskongresse}

\section{Bremen}

\section{Jahreskongress der GTH}

18.-21. Februar 2020

\section{Bremen}

Kongresspräsidenten:

Prof. Dr. Florian Langer

Prof. Dr. Thomas Renné

In Kürze finden Sie hier

die ersten Informationen:

www.gth2020.org

\section{Lausanne}

65. Jahreskongress der GTH

23.-26. Februar 2021

\section{Lausanne}

Kongresspräsident:

Prof. Dr. Lorenzo Alberio

\section{Sachsen}

\section{Jahreskongress der GTH}

Februar 2022

\section{Sachsen}

Kongresspräsidenten:

Dr. Ute Scholz

PD Dr. Jan Beyer-Westendorf 


\section{GTH Highlights 2019:}

Ein Highlight für die GTH
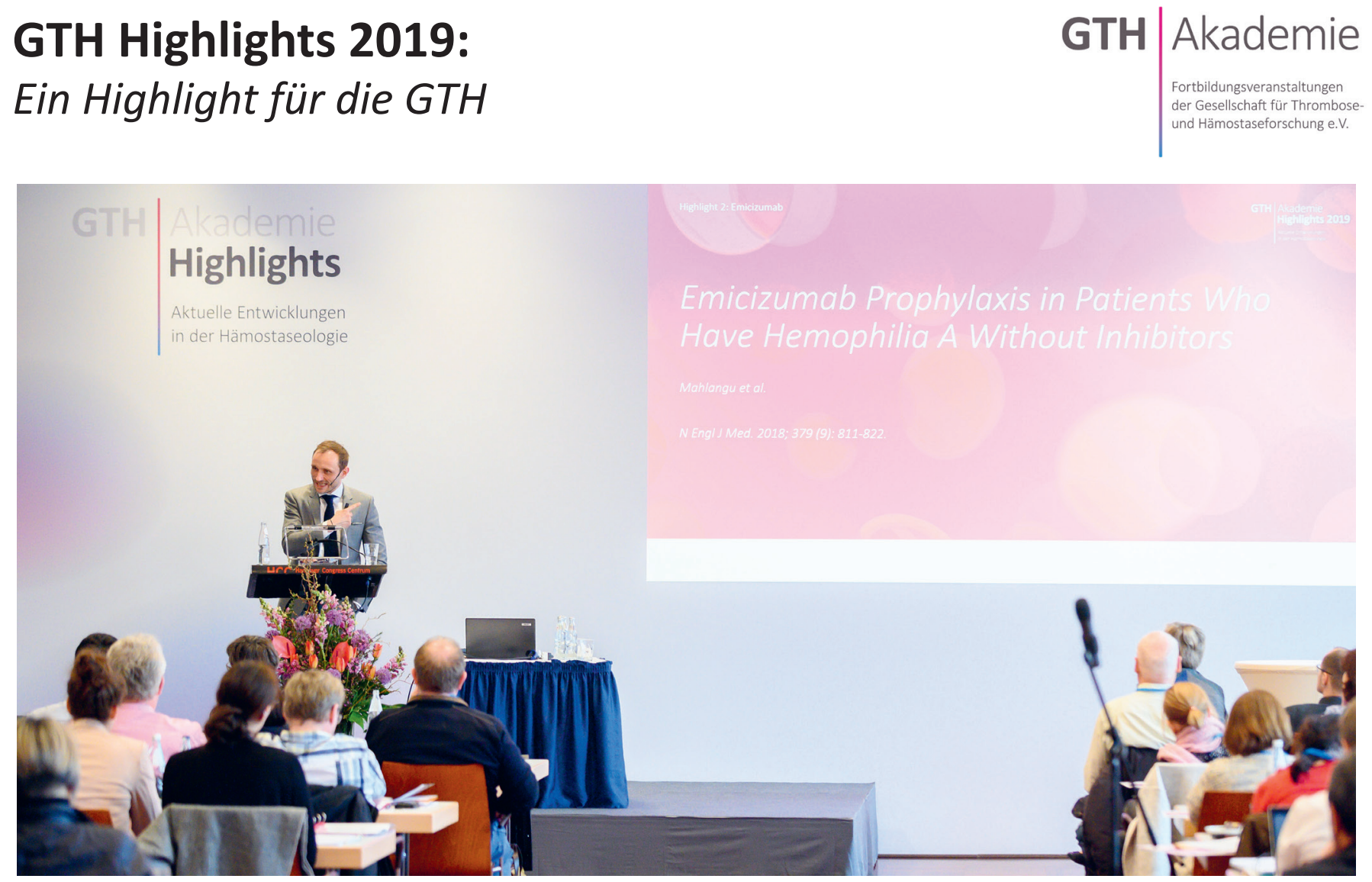

Am 15 und 16. März fanden im Rahmen der GTH Akademie die „Highlights - Aktuelle Entwicklungen in der Hämostaseologie" im HCC in Hannover statt. Nach intensiver Planung und Vorbereitung durch das Steering Committee unter Leitung von Prof. Andreas Tiede und die Geschäftsstelle der GTH konnte die zweitägige, hochkarätige Fortbildungsveranstaltung mit begleitender Industrieausstellung und Symposien alle Teilnehmerplätze als ausgebucht vermelden und startete freitags pünktlich und reibungslos nach dem Begrüßungsimbiss um $11.30 \mathrm{Uhr}$.

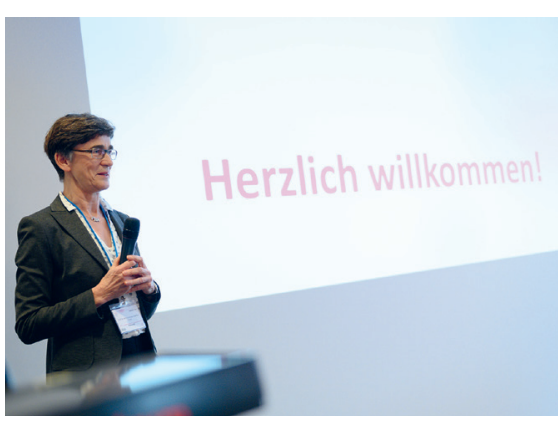

In dem bis auf den letzten Platz gefüllten „Roter Saal“ des HCC in Hannover eröffnete Bettina Kemkes-Matthes die Premiere der GTH Highlights. Hannover hat als Standort eine besondere Historie für die GTH: Hier fand vor 24 Jahren der Intensivkurs unter Leitung von Monika Barthels statt, die inzwischen seit 24 Jahren die etablierteste unter den Fortbildungen der GTH, die nun auch Teil

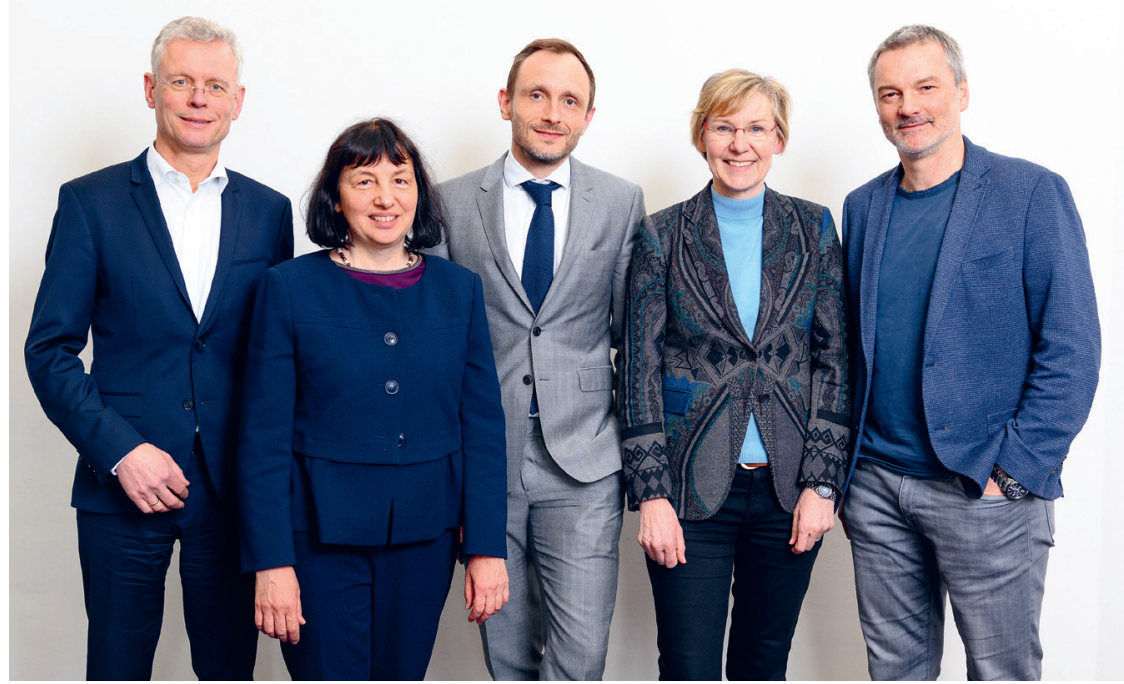

Das Steering Committe der GTH Highlights, von links nach rechts: Prof. Dr. Andreas Greinacher, Prof. Dr. Barbara Zieger, Prof. Dr. Andreas Tiede, Prof. Dr. Ingrid Pabinger, PD Dr. Jan Beyer-Westendorf. 
der neu gegründeten GTH Akademie ist. Hierzu gehört auch der Laborkurs unter Leitung von Ute Scholz, der weiterhin in Leipzig stattfindet. Als dritte Fortbildung im Rahmen der Akademie beschloss der Vorstand 2018, unter wissenschaftlicher Leitung von Andreas Tiede die „Highlights" aus der Taufe zu heben. Entspre-

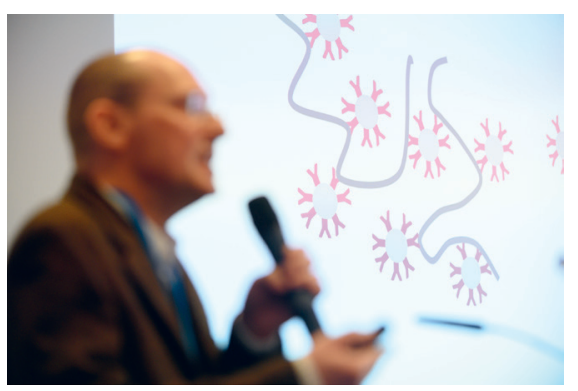

chend ging der besondere Dank von Frau Kemkes-Matthes an Herrn Tiede und das Steering Committee.

Herr Tiede freute sich in seiner Begrüßung besonders, als „Wahl-Hannoveraner" die GTH Highlights in Hannover ausrichten zu dürfen. Sein Dank ging an Bettina Kemkes-Matthes für die Unterstützung und das Vertrauen des Vorstands, an das Steering Committee für die hervorragende fachliche Arbeit und an die GTH Geschäftsstelle für das geleistete „Gesellenstück" in der Planung und Organisation der Veranstaltung.

Zwei Tage präsentierten die Referenten den Anwesenden "State of the Art" und „Highlights" aus der Hämostaseologie
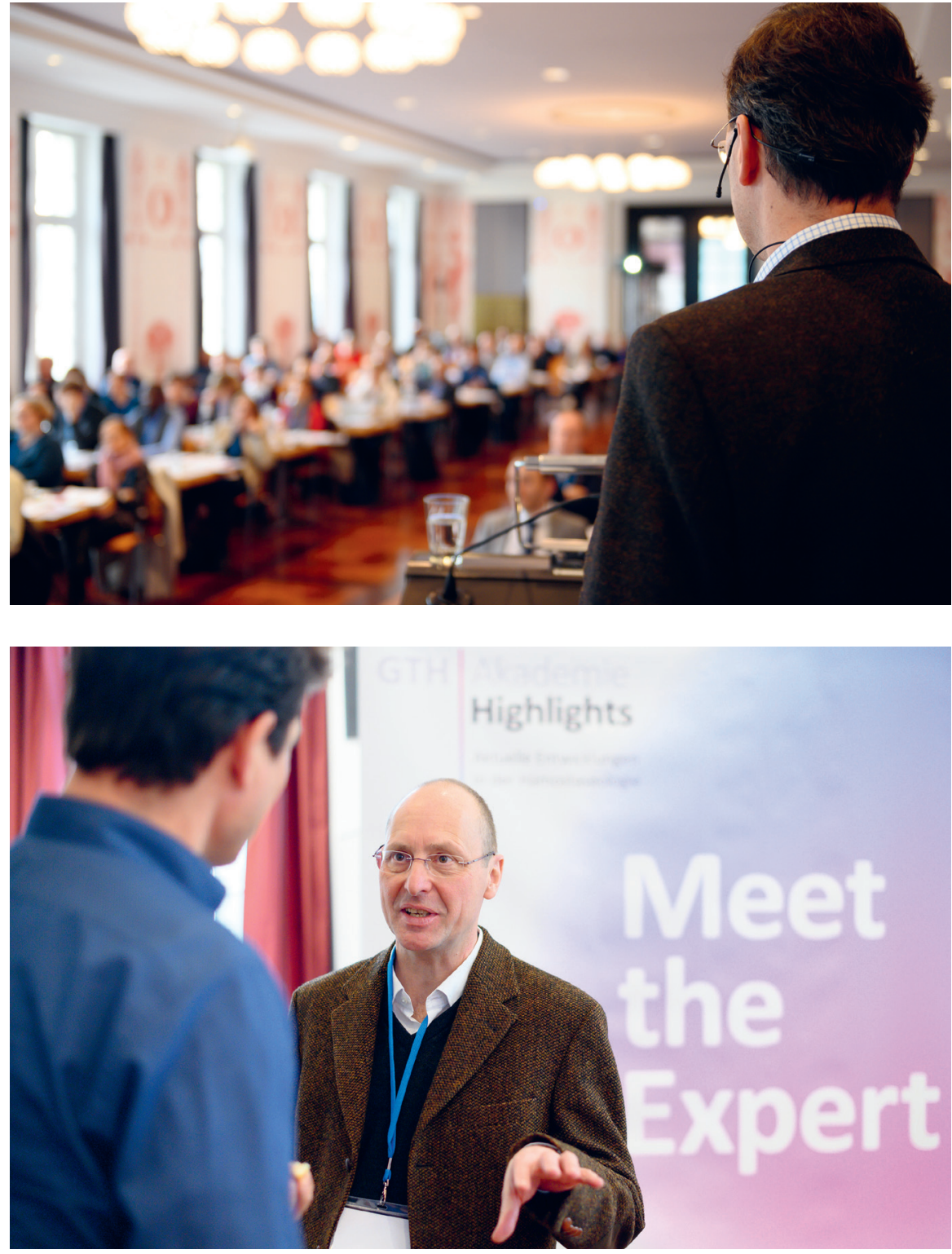

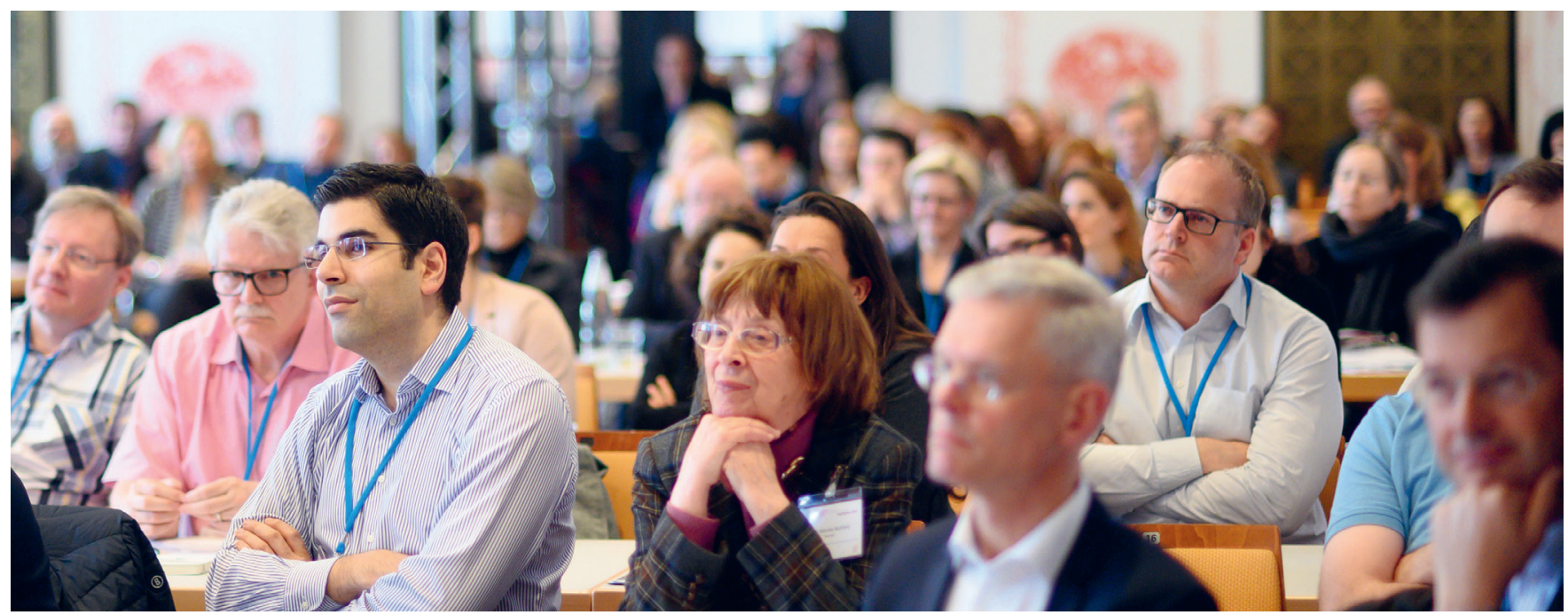


auf höchstem Niveau. Die nach den Vorträgen und am „Meet the Expert“ Stand angebotenen Gesprächs- und Diskussionsmöglichkeiten wurden intensiv genutzt, hier standen die Experten gerne und ausgiebig für Fragen zur Verfügung. Das Ziel der neuen Fortbildung, den Teilnehmern verschiedener Fachgebiete in komprimierter Form das aktuelle Wissen der Hämostaseologie zur Anwendung in der Praxis vermitteln zu können, wurde mehr als erreicht.

\section{"Die Organisation und Themen der Highlights haben mir sehr gut gefallen. Insbesondere der Aufbau der Vorträge, in denen zunächst die Basics und anschließend die "Highlights" vermittelt wurden." \\ Dr. Nicole Hartmann, Teilnehmerin}

Die Teilnehmer erhielten zu Veranstaltungsbeginn Tagungsordner mit allen Manuskripten der Fachvorträge in gedruckter Form, zuvor wurden bereits Mails mit individuellen Zugangscodes für den Download der Referate als PDF oder eBook versandt.

Die im Rahmenprogramm stattfindende Industrieausstellung sowie die drei Symposien der Sponsoren komplettierten das Angebot, so dass die "Highlights“ zu einer rundum gelungenen Veranstaltung wurden.

„Wie Thomas Renné ganz neue Forschungsergebnisse lebhaft, praxisnah und voller Enthusiasmus präsentierte, war wirklich beeindruckend und eine wunderbare Ergänzung zu den klinischen Beiträgen zu State-of-theArt und aktuellen Highlights! An die Spülmaschinen-Tabs werden sich viele von uns erinnern, wenn wir an Polyphosphate auf Thrombozyten denken."

Prof. Dr. Daniel Dürschmied, Referent

"Ich bin viele gute Veranstaltungen gewöhnt, diese hat das noch getoppt: Die Vorträge waren „allererste Sahne", was ich in der Form nicht erwartet habe. Ich werde mit sicherheit wiederkommen."

Dr. Thomas Lang, Teilnehmer

Die Erwartungen der Gäste erfüllten sich in jeder Hinsicht: In der Evaluation wurden die Vorträge durchgehend im obersten Bereich bewertet. Themenauswahl, Zeitplan und Organisation erhielten ebenfalls meist die volle Punktzahl. Das HCC als Location fand großen Anklang: die familiäre „Universitätsatmosphäre“ wurde als sehr angenehm empfunden. Fast alle Teilnehmer möchten 2020 wieder dabei sein.

\begin{abstract}
"Als Hannoveranerin waren die Highlights für mich sozusagen ein Heimspiel! Vom Thema Blutungsneigung über Thrombose fassten Experten alle aktuellen Highlights sehr gut zusammen. Besonders zu erwähnen sind die familiäre Atmosphäre, die guten Diskussionen im kleinen Kreis - auch mit Ärzten aus anderen Fachgebieten - und die angenehme Organisation der gesamten Veranstaltung."
\end{abstract}

Yvonne Wieland, Teilnehmerin

Abschließend bedankte sich Johannes Oldenburg als neuer Vorsitzender der GTH bei den Organisatoren und beglückwünschte Herrn Tiede zum Start der GTH Highlights. Besonders betonte er zum Auftakt der GTH Akademie die Aufgabe der GTH zur Ausbildung und Förderung des Nachwuchses. Andreas Tiede bedankte sich bei allen Referenten und Helfern sowie den Sponsoren für deren Engagement, das dem neuen Flaggschiff der GTH einen so guten Start ermöglichte.

\section{Save the date}

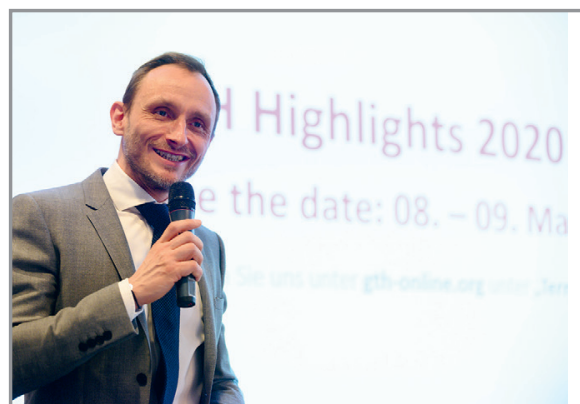

Der Termin für die GTH Highlights 2020 steht bereits fest:

\section{8. - 09. Mai 2020 \\ HCC Hannover}

Weitere Informationen finden Sie bald auf der Webseite.

Wir informieren Sie, sobald unser Anmeldeportal freigeschaltet ist. www.gth-highlights.org 
12. - 15. Juni, Wilsede

Jahrestagung des

AK Hämostaseologie AssistentInnen und Basiskurs „Thrombophilie“

www.hämostaseologie-assistentinnen.org

13. Juni - 14. Juni, Düsseldorf

1. Nationaler Kongress zur kritischen Extremitätenischämie

Wissenschaftliche Leitung:

Dr. Michael Lichtenberg

Prof. Dr. Markus Steinbauer

Prof. Dr. Giovanni Torsello

Prof. Dr. Thomas Zeller

Veranstalter:

Kongress- und MesseBüro

Lentzsch $\mathrm{GmbH}$

Tel.: 06172 / 6796-0

info@kmb-lentzsch.de

www.cli-kongress.de

20. - 22. Juni, Wien

Angiologische Sommerakademie Wien 2019 und Jahrestagung der Österreichischen Gesellschaft für internistische Angiologie (ÖGIA)

Veranstalter:

Tagungsmanagement Ursula

Partsch-Brokke

Tel.: +43 / 2672 / 88996

www.vascular-summer-academy.info vasc/index.php

25. - 28. Juni , Amsterdam

15th International Conference on

SubArachnoid Hemorrhage

Veranstalter:

Kenes M+

General Inquiries:

Ms. Merve Tufekci

mtufekci@kenes.com

www.isah-conference.org
31. August, Berlin

23. Symposium Aktuelles zur Thrombose und Blutung

Wissenschaftliche Leitung:

Priv.-Doz. Dr. med. J. Koscielny Veranstalter:

Charité Healthcare Services $\mathrm{GmbH}$

Tel.: 030 / 450578206

Mail: christine.thol@charite.de

\section{6.-7. September, Frankfurt a.M.}

10. Frankfurter Gerinnungssymposium

Wissenschaftliche Leitung:

Prof. Dr. Edelgard Lindhoff-Last

Prof. Dr. Rupert M Bauersachs

Prof. Dr. Viola Hach-Wunderle

Veranstalter:

Kongress- und MesseBüro

Lentzsch $\mathrm{GmbH}$

Tel.: 06172 / 6796-0

info@kmb-lentzsch.de

www.gerinnungssymposium-frankfurt.de

12.-14. September, Leipzig

3. DGA - Interventionskongress und

48. Jahrestagung der Deutschen Gesellschaft für Angiologie -

Gesellschaft für Gefäßmedizin e.v.

Wissenschaftliche Leitung:

Prof. Dr. Dierk Scheinert

PD Dr. med. Andrej Schmidt

Dr. med. Katja S. Mühlberg

Kongressorganisation:

Kongress- und MesseBüro

Lentzsch $\mathrm{GmbH}$

Tel.: 06172 / 6796-0

info@kmb-lentzsch.de

www.angiologie2019.de
12.-14. September, Leipzig

GTH Akademie:

12. Laborkurs -

Methoden der Hämostaseologie

Wissenschaftliche Leitung:

Dr. Ute Scholz

Veranstalter:

GTH-Gesellschaft für Thrombose-

und Hämostaseforschung e.V.

in Zusammenarbeit mit

BFSH e.V.- Bündnis zur Förderung

der Sicherheit von Hämophilen

Tel.: 0361 / 6638260

Mail: kontakt@bfsh.info

www.gth-laborkurs.org

02. November, Hamburg

9. Eppendorfer Gerinnungssymposium

Wissenschaftliche Leitung:

Prof. Dr. Florian Langer

Dr. Katharina Holstein

11. - 15. November, Giessen

GTH Akademie:

24. Intensivkurs -

Einstieg in die Hämostaseologie

Wissenschaftliche Leitung:

Prof. Dr. Bettina Kemkes-Matthes

Veranstalter:

GTH- Gesellschaft für Thrombose-

und Hämostaseforschung e.V.

Organisation und und Anmeldung:

Susanne Rossi

gth.crashkurs@innere.med.uni-giessen.de www.gth-intensivkurs.org

\section{Zertifizierung der Hämophilie-Zentren: Auditorinnen und Auditoren gesucht!}

Herr Prof. Dr. Hermann Eichler von der Arbeitsgruppe "Zertifizierung der Hämophilie-Zentren" hat vom GTH-Vorstand den Auftrag erhalten, dieses Projekt zu realisieren. Die Leitlinie dazu wurde bereits in der HÄMOSTASEOLOGIE veröffentlicht und ist ebenfalls auf der Webseite der GTH zu finden.

Die Zertifizierung wird in Zusammenarbeit mit einem professionellen externen Partner durchgeführt, nach Möglichkeit sollen die ersten Audits vor Ort noch in diesem Jahr erfolgen. Dafür benötigen wir ca. 20 Experten aus dem Fachbereich, die vor dem ersten Einsatz als Auditorin oder Auditor beim externen Partner geschult werden.

Für die paritätische Besetzung des Auditorenteams suchen wir erfahrene und motivierte Kolleginnen und Kollegen aus Kliniken, Universitäten und Praxen Deutschlands, Österreichs und der Schweiz.
Haben Sie Interesse an der Mitarbeit am Projekt „Zertifizierung der Hämophilie-Zentren" und möchten Sie Auditor/in werden?

Bitte melden Sie sich bis zum 30. Juni 2019 per Mail in der GTH-Geschäftsstelle in Köln, dort erhalten Sie weitere Informationen:

GTH Geschäftsstelle mail@gth-online.org 